[Article]

\title{
石墨烯的纳米摩擦与磨损性质
}

\author{
朱齐荣 ${ }^{1}$ 李慧琴 ${ }^{1}$ 李 宁 ${ }^{1}$ 柴 静 $^{2}$ 高润纲 ${ }^{2}$ 梁 齐 ${ }^{1, *}$ \\ ( ${ }^{1}$ 上海交通大学分析测试中心, 上海 $200240 ;{ }^{2}$ 上海交通大学微纳科学技术研究院, 上海 200240)
}

\begin{abstract}
摘要: 采用乙醇溶剂剥离的方法制备石墨烯. 通过对溶剂温度、超声时间、超声功率和溶剂离心速度及时间的 控制, 从高定向热解石墨(HOPG)制备得到少层石墨烯. 用原子力显微镜(AFM)研究了云母基底上不同层数石 墨烯在真空中的纳米摩擦过程, 发现从约 4 个原子单层 $(4 \mathrm{ML})$ 起, 摩擦系数基本不再变化, 但摩擦力仍随着厚 度的增加而显著减小, $7 \mathrm{ML}$ 之后, 其摩擦系数基本接近于零. 在磨损实验中, 少层石墨烯表面存在刮坏的现象, 且不同厚度的石墨烯的磨损现象明显不同, 其中 $2 \mathrm{ML}$ 石墨烯相比 $4 \mathrm{ML}$ 石墨烯表现出较好的耐磨损性能, 且不 具有摩擦方向依赖性. 测试了真空下少层石墨烯和云母表面的粘附力, 发现不同层厚的石墨烯相差不大, 因此 认为基底效应并不是磨损性质差异的主要原因. 相对于单层石墨烯, 少层石墨烯在抗磨损涂层等领域有着很大 的潜在应用价值.
\end{abstract}

关键词: 高定向热解石墨; 石墨烯; 纳米摩擦学; 原子力显微镜; 磨损 中图分类号: 0647

\section{Nanotribological and Wear Properties of Graphene}

\author{
ZHU Qi-Rong ${ }^{1} \quad$ LI Hui-Qin ${ }^{1} \quad$ LI Ning ${ }^{1} \quad$ CHAl Jing ${ }^{2} \quad$ GAO Run-Gang ${ }^{2} \quad$ LIANG Q1',* \\ ('Instrumental Analysis Center, Shanghai Jiao Tong University, Shanghai 200240, P. R. China; \\ ${ }^{2}$ Research Institute of Micro/Nano Science and Technology, Shanghai Jiao Tong University, Shanghai 200240, P. R. China)
}

\begin{abstract}
We prepared few-layer graphene samples by liquid-phase exfoliation in ethanol. By controlling the solvent temperature, sonication time and power, and centrifugation speed and time, we fabricated several-layer graphene from highly oriented pyrolytic graphite (HOPG). The obtained supernatant was added dropwise onto freshly cleaved mica surfaces. Nanotribological study of the samples under high vacuum by atomic force microscope (AFM) showed that frictional force decreased as the number of monolayers (ML) of graphene increased, and their frictional coefficient remained constant when the sample was thicker than about $4 \mathrm{ML}$. When the coverage reached $7 \mathrm{ML}$, the frictional coefficient was close to zero. In wear experiments, 2-ML graphene exhibited better wear resistance than the 4-ML sample and had no dependence on directional friction. We also measured the adhesion force of samples containing different numbers of layers of graphene and the mica surface, and found that substrate adhesion is not the main reason for the wear resistance properties of 2-ML graphene. Compared with single-layer graphene, the low friction coefficient of few-layer graphene makes it promising for application in areas such as data storage devices, nanoelectromechanical systems, and anti-wear coatings.
\end{abstract}

Key Words: Highly oriented pyrolytic graphite; Graphene; Nanotribology; Atomic force microscope; Wear

Received: January 29, 2013; Revised: May 2, 2013; Published on Web: May 3, 2013.

"Corresponding author. Email: qiliang@sjtu.edu.cn; Tel: +86-21-34205698.

The project was supported by the National Natural Science Foundation of China (10974134).

国家自然科学基金(10974134)资助项目

(C) Editorial office of Acta Physico-Chimica Sinica 


\section{1 引言}

近 20 年以来, 碳纳米材料一直是科技创新的热 点, 从富勒烯到碳纳米管再到今天的石墨烯, 无一 不掀起了科学界的研究热潮. 石墨烯是一种有单层 碳原子紧密堆积成二维蜂窝状晶格结构的碳质新 材料. 自 2004 年 Geim 等 ${ }^{1}$ 首次用微机械剥离法成功 获得单层的石墨烯以来, 其特有的电学、热学、力学 等性质引起了科学家的广泛关注..$^{2-6}$ 随着研究的深 入展开, 石墨烯的制备方法也越来越多样化, 目前 主要的方法有微机械剥离法、 ${ }^{1}$ 氧化还原法、 ${ }^{7}$ 溶剂 剥离法、 ${ }^{8}$ 化学气相沉积法 ${ }^{9,10}$ 和外延生长法 ${ }^{11}$ 等. 由 于石墨烯超薄的厚度及优异的摩擦性能, 使其在纳 米尺寸数据存储设备、纳米复合材料和纳米机电系 统中具有很大的潜在应用价值. ${ }^{12}$ 这就使得石墨烯 与其它材料接触时表面的相互作用研究, 如摩擦 力、粘附力和磨损等, 显得尤为重要.

无论是在真空环境还是大气环境下, 大多数纳 米摩擦领域研究显示, 相对于单层石墨烯, 多层石 墨烯表现出更低的摩擦力及摩擦系数. 对于这种现 象的解释至今尚未得到最终统一, 解释的原理包括 皱褶效应及电子-声子耦合效应等. ${ }^{13-16}$ 这种不一致 的解释, 与研究者的制备方法、石墨烯的基底及测 试条件有很大的关系. 然而在这些石墨烯的摩擦学 研究中, 却较少提及所可能存在的磨损问题. ${ }^{17-19}$ 为 了考察石墨烯在纳米尺寸数据存储设备、纳米复合 材料和微纳机电系统(MEMS)中的实际应用, 以及 其是否适合作为固体润滑剂, 石墨烯磨损的研究至 关重要.

本文采用溶剂剥离法将从高定向热解石墨 (HOPG)上剥离下的石墨片放到无水乙醇中超声, 离 心后, 将制备好的石墨烯乙醇溶液滴在干净的新解 理云母片上, 密封条件下自然干燥. 在高真空中, 将 制备好的样品用原子力探针显微镜(接触模式 AFM)进行检测, 研究了不同层数石墨烯的摩擦力 及磨损过程, 以及在真空中改变不同扫描角度后, 石墨烯形貌的变化.

\section{2 实验部分}

\section{1 石墨烯的制备}

实验采用昆山市超声仪器有限公司生产的数 控超声波清洗器(型号 KQ-500DE), 离心机为上海 卢湘仪 TG16-WS 台式高速离心机, 高定向热解石墨 是购于上海奈米派实业有限公司的高序石墨(型号
GRAS/1.2), 无水乙醇(纯度是 99.7\%) 是常熟市杨园 化工有限公司生产的. 实验过程如下, 首先用特定 的胶带从 HOPG 上撕下石墨片层, 将撕下的石墨称 取约 $0.002 \mathrm{~g}$ 加到盛有 $60 \mathrm{~mL}$ 无水乙醇的烧杯中, 密 封后放入超声波清洗器中超声. 在超声过程中, 温 度保持在 $20-25^{\circ} \mathrm{C}$, 超声功率为 $200 \mathrm{~W}$. 超声约 $48 \mathrm{~h}$ 之后, 将石墨烯溶液在 $10000 \mathrm{r} \cdot \mathrm{min}^{-1}$ 下离心 $5 \mathrm{~min}$. 离心之后, 取上层清液滴到新解理的云母表面上, 密封环境下室温干燥, 干燥后放到原子力探针显微 镜中抽至高真空, 为后面的测试实验做准备. 为了 证实少层石墨烯的存在与否, 将离心后的上层清液 滴到 $\mathrm{SiO}_{2}$ 基底上, 在干燥箱 $\left(60^{\circ} \mathrm{C}\right)$ 中烘干后, 用德 国 Bruker Senterra R200-L 型显微激光拉曼光谱仪 测试得到拉曼光谱.

\section{2 石墨烯的摩擦学及其磨损性质测试}

摩擦学性能测试采用日本精工公司生产的环 境可控型多功能扫描探针显微镜(型号 SII NanoNavi E-Sweep)进行, 测试石墨烯纳米摩擦学采用探 针为日本 OLYMPUS 生产的 $\mathrm{Si}_{3} \mathrm{~N}_{4}$ 探针, 其法向弹性 系数为 $\sim 0.08 \mathrm{~N} \cdot \mathrm{m}^{-1}$, 悬臂长为 $200 \mu \mathrm{m}$; 测试磨损的 探针是日本 Veeco 公司生产的 $\mathrm{Si}_{3} \mathrm{~N}_{4}$ 探针, 其法向弹 性系数为 $0.58 \mathrm{~N} \cdot \mathrm{m}^{-1}$, 悬臂长为 $100 \mu \mathrm{m}$. 纳米摩擦 学研究的实验过程中, 腔体的真空度始终保持在 $1.5 \times 10^{-4}-3.6 \times 10^{-4} \mathrm{~Pa}$ 之间, 扫描速率为 $1.5 \mathrm{~Hz}$, 扫描 范围约为 $60 \mathrm{~nm} \times 60 \mathrm{~nm}$, 扫描角度为 $90^{\circ}$, 由于并未 对横向扭力系数作数值标定, 这里摩擦力的坐标用 任意单位表示; 磨损实验的真空度始终保持在 $4 \times$ $10^{-4}-7 \times 10^{-4} \mathrm{~Pa}$ 之间, 扫描速度为 $1.5 \mathrm{~Hz}$, 扫描范围 是 $8.4 \mu \mathrm{m} \times 8.4 \mu \mathrm{m}$. 在 AFM 接触模式下, 测量石墨烯 形貌磨损的整个变化过程, 并改变不同的扫描角 度, 研究扫描方向对磨损的影响情况. 此外, 我们还 分析了不同层厚的石墨烯和云母表面与 $\mathrm{Si}_{3} \mathrm{~N}_{4}$ 探针 之间的粘附力.

\section{3 结果与讨论 \\ 3.1 石墨烯膜的拉曼光谱}

图 1 为所用样品的激光拉曼光谱图. 其测试条 件是在室温下进行, 入射功率为 $20 \mathrm{~mW}$, 激光波长 为 $532 \mathrm{~nm}$, 曝光时间为 $40 \mathrm{~s}$. 从图中可以见到 $D$ 峰在 $1374 \mathrm{~cm}^{-1}$ 附近, $G$ 峰在 $1577 \mathrm{~cm}^{-1}$ 附近, $2 D$ 峰在 2894 $\mathrm{cm}^{-1}$ 附近. 根据以上峰位数据, 可以确定在所制备的 上层清液中的片层就是石墨烯. ${ }^{20}$

\section{2 真空中石墨烯的纳米摩擦性质}




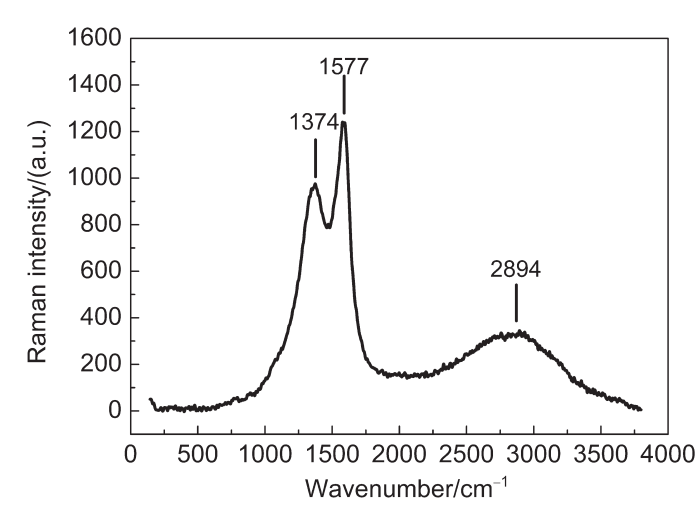

图 1 大量少层石墨烯的拉曼谱图

Fig.1 Raman spectrum of numerous graphene in several layers

图 2 是云母表面不同厚度石墨烯薄层 $(3 、 4 、 7 、$ $13 \mathrm{ML})$ 的摩擦力曲线. 由于未对所使用的探针进行 横向扭转系数的标定, 我们采用归一化的任意单位 作为摩擦力的坐标. 从图 2 中可以看出, 随着层数的 增加, 摩擦力逐步降低. 从拟合的曲线中可以看出 从 4 ML之后, 摩擦力仍在进一步降低, 而摩擦系数 (斜率)不再有明显变化. 特别值得注意的是, 当石墨 烯的厚度达到 $7 \mathrm{ML}$ 以上时, 在小载荷 $(10 \mathrm{nN}$ 以内) 情况下, 其摩擦系数接近于零, 且摩擦力在一定实 验误差范围内不再变化, 这种特殊的摩擦性质在微 纳机电系统中有着重要的应用前景. 以上的摩擦力 曲线均是在相同的扫描面积、扫描速率及温度下测 得的, 排除了外界因素的影响. 由于表面粘附力是 影响纳米摩擦性质的重要因素, 因此, 我们分别测 量了这几个不同厚度石墨烯以及清洁云母的表面 粘附力, 发现在误差范围内数值上并无明显区别. 综观之前一系列的研究, Filleter 等 ${ }^{14}$ 发现在 $\mathrm{SiC}$ 基底 上双层石墨烯的摩擦力及摩擦系数小于单层石墨 烯. Lee等 ${ }^{13}$ 发现在 $\mathrm{SiO}_{2} / \mathrm{Si}$ 基底上不同层数石墨烯 的摩擦力及摩擦系数随着层数的增加而减小, 到 4 ML之后, 摩擦力及摩擦系数几乎就没有太大差异, 且不同层数石墨烯的粘附力不可区分. 另外 Lee等 ${ }^{13}$ 还提到云母基底对上述现象有抑制的作用, 但是我 们在真空中的实验证明了尽管有一定抑制作用, 但 是这一摩擦性质仍然是存在的. 这样也说明了无论 是 $\mathrm{SiC}$ 基底、 $\mathrm{SiO}_{2} / \mathrm{Si}$ 基底或云母基底, 石墨烯的摩擦 力随着层数的增加而减小, 且摩擦系数也相应减 小, 到 7 ML之后几乎为零. 这种现象是由于随着层 数的增加, 针尖与祄底之间较强的相互作用力逐渐 被石墨烯的覆盖层屏蔽, 即 van der Waals 力的作用 减弱, Lennard-Jones 势的吸引能以距离的负 6 次方

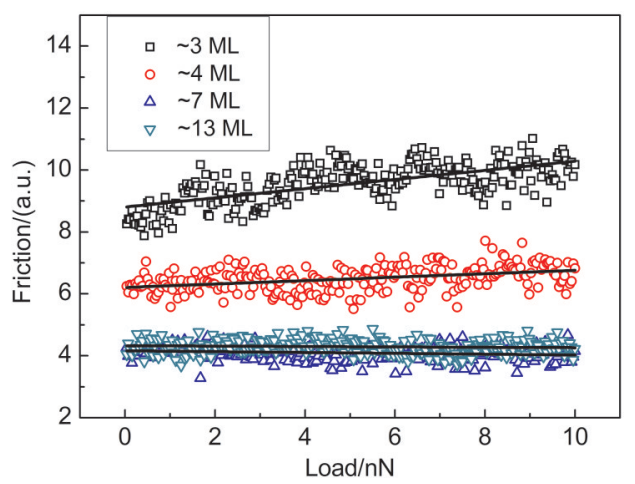

图 2 云母上不同层数石墨烯的摩擦力对载荷关系图 Fig.2 Friction versus load curves for graphene films with different layers on mica

衰减, 也就是说随距离的衰减极快. 大约 7 层石墨烯 层后祄底的作用基本可以忽略, 相应的摩擦力也就 在一定的误差范围内不再变化.

\section{3 真空中样品的磨损}

为了防止大气中的水蒸气等吸附物在表面形 成的水膜所产生的毛细力影响实验结果, 我们将磨 损实验放入高真空中进行研究. 在实验的扫描过程 中针尖始终保持着对样品表面不超过 $1 \mathrm{nN}$ 的正压 力. 图 3 是真空环境下吸附在清洁云母表面的多层 石墨烯逐渐磨损过程的 AFM 形貌图. 图 3(a-f)分别 对应着第 2、3、4、5、8、20 次扫描后得到的 AFM 形貌 图, 其中图 3(g)和 3(h) 分别是图 3(b) 和 3(f) 标记中的 剖线图. 另外, 由于石墨分子层的理论层间距约为 $0.34 \mathrm{~nm},{ }^{1}$ 从图 $3(\mathrm{~g})$ 可以看出约 $0.6 \mathrm{~nm}$ 和约 $1.3 \mathrm{~nm}$ 石 墨烯的存在, 它们分别对应着 2 ML和 4 ML 石墨 烯; 图 3(h)中均为 $\sim 0.7 \mathrm{~nm}$ 的石墨烯, 即 2 ML 石墨 烯. 从图 3(b) 和 3(g) 可以看出在磨损发生之前及磨 损前期, 石墨烯为 2 ML 层和 4 ML 层的组合; 从图 3(f)和 3(h) 可以看出磨损后期, 4 ML 结构的石墨烯 几乎磨损殆尽, 但是 2 ML 结构的石墨烯几乎完好 无损. 在 AFM 图扫描次数达到 8 次后, 其磨损状况 基本达到了稳定, 形貌图已经没有太大变化, 之后 又进行了二十余次扫描也没有大的磨损的发现. 整 个实验过程采用相同的扫描速率和扫描角度(方 向), 不同于大气环境下的石墨烯, 在真空中样品表 面包括水分子在内的气体吸附分子显著减少, 样品 表面的毛细作用力基本可以忽略弱化. 另外, 不同 于大多数文章中将石墨烯放置到 $\mathrm{SiO}_{2}$ 或 $\mathrm{SiC}$ 基底的 研究, 我们将石墨烯置于能达到原子级平坦的新鲜 清洁云母片上, 这样就可以在一定程度上抑制石墨 烯的褶皱效应对摩擦力及磨损的影响. ${ }^{21,22}$ 以上的现 

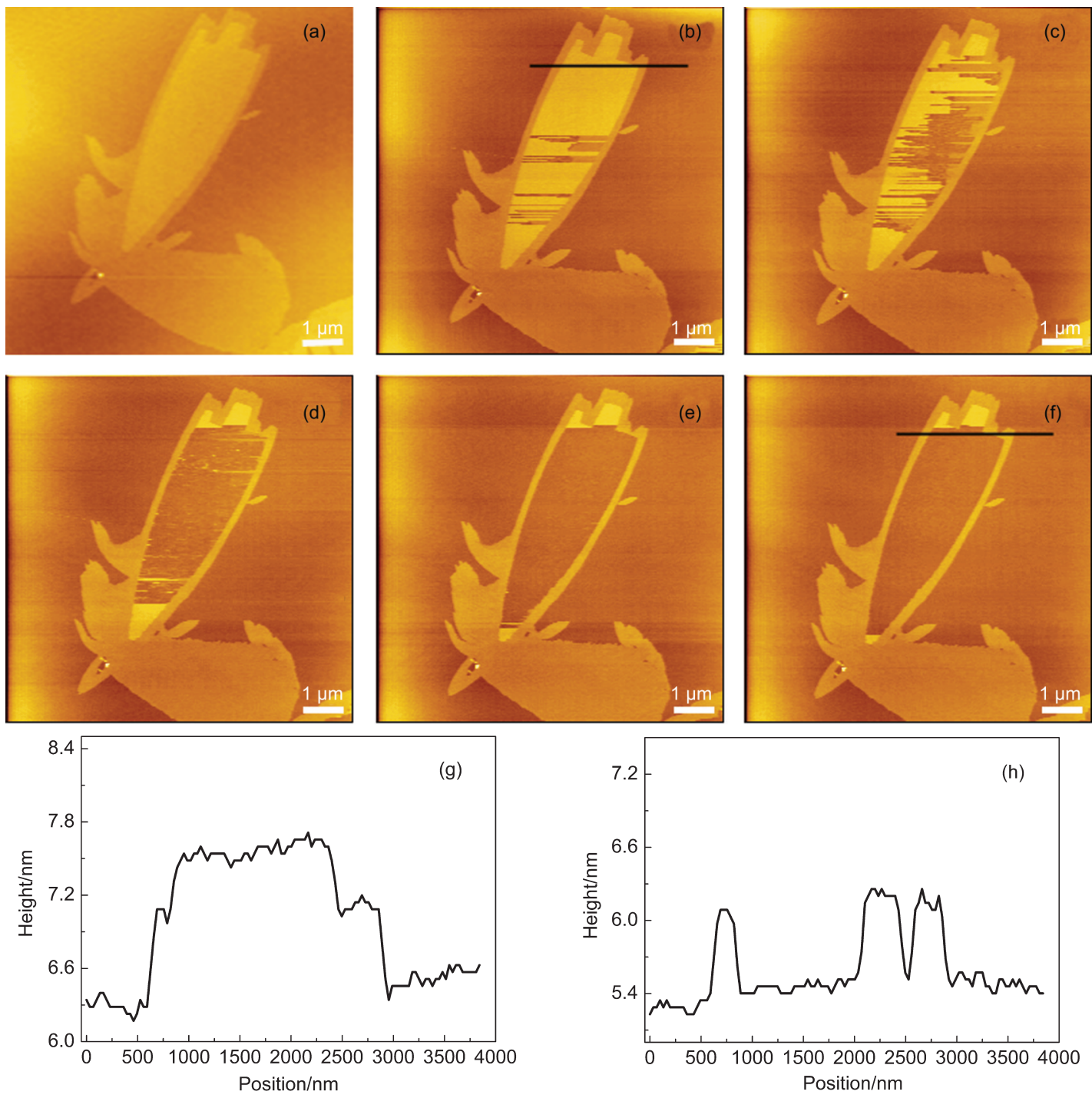

图 3 云母上石墨烯磨损过程的 AFM 形貌图

Fig.3 AFM topographic images of the wear process of graphene on mica

From (a) to (f), the numbers of scans are 2, 3, 4, 5, 8, and 20, respectively. (g) and (h) are the line profiles of the marking parts in (b) and (f), respectively. The rotation of the images above is $0^{\circ}$.

象说明, 尽管 4 ML 石墨烯比 2 ML 石墨烯拥有更 低的摩擦力, 但是 $2 \mathrm{ML}$ 石墨烯拥有更大的耐磨损 性能. 我们推测, 这可能是由于分子层数少, 石墨烯 覆盖层与云母衬底表面分子相互作用更强的缘 故. ${ }^{13}$ 在研究表面科学中, 基底的效应一向是不可忽 略的, 23,24 随着样品表面与基底的距离增大, 石墨烯 与云母基底之间的相互作用力则相应变弱, 因此在 相同的针尖压力下, 4 ML 结构的石墨烯比 2 ML 结构更加容易被针尖破坏并带走.

相对于其它材料, 2 ML 石墨烯已经拥有很低 摩擦力, 与 $\sim 4 \mathrm{ML}$ 石墨烯及石墨相差不是很大, 且具 有更好的抗磨性. 这个实验现象说明, 很可能 2 ML 石墨烯更适合作为纳米机电系统等领域的固体润
滑薄膜.

\section{4 真空中不同扫描方向下石墨烯的磨损}

为了进一步验证我们的结论, 考虑到石墨烯平 面结构的对称性, 我们改变了不同的扫描方向, 以 研究其磨损性质与原子排列方向的依赖性和关联 性. 图 4 是在图 3 的基础上改变不同扫描角度时石 墨烯样品的AFM形貌图. 任选其中两个角度看表面 的石墨烯片层厚度变化, 由图 4(e)和 4(f)发现图 4(a) 和 4(c)中的石墨烯厚度均为 $0.7 \mathrm{~nm}$, 即两个石墨烯 单层的厚度. 我们尝试变换了四个角度, 分别为 $90^{\circ} 、-90^{\circ} 、-120^{\circ}$ 和 $-60^{\circ}$, 且扫描次数分别达到 22 、 $23 、 27 、 33$ 次, 样品表面的磨损变化不大, 特别是 2 ML 石墨烯依然几乎无损坏现象. 这种现象, 表明即 

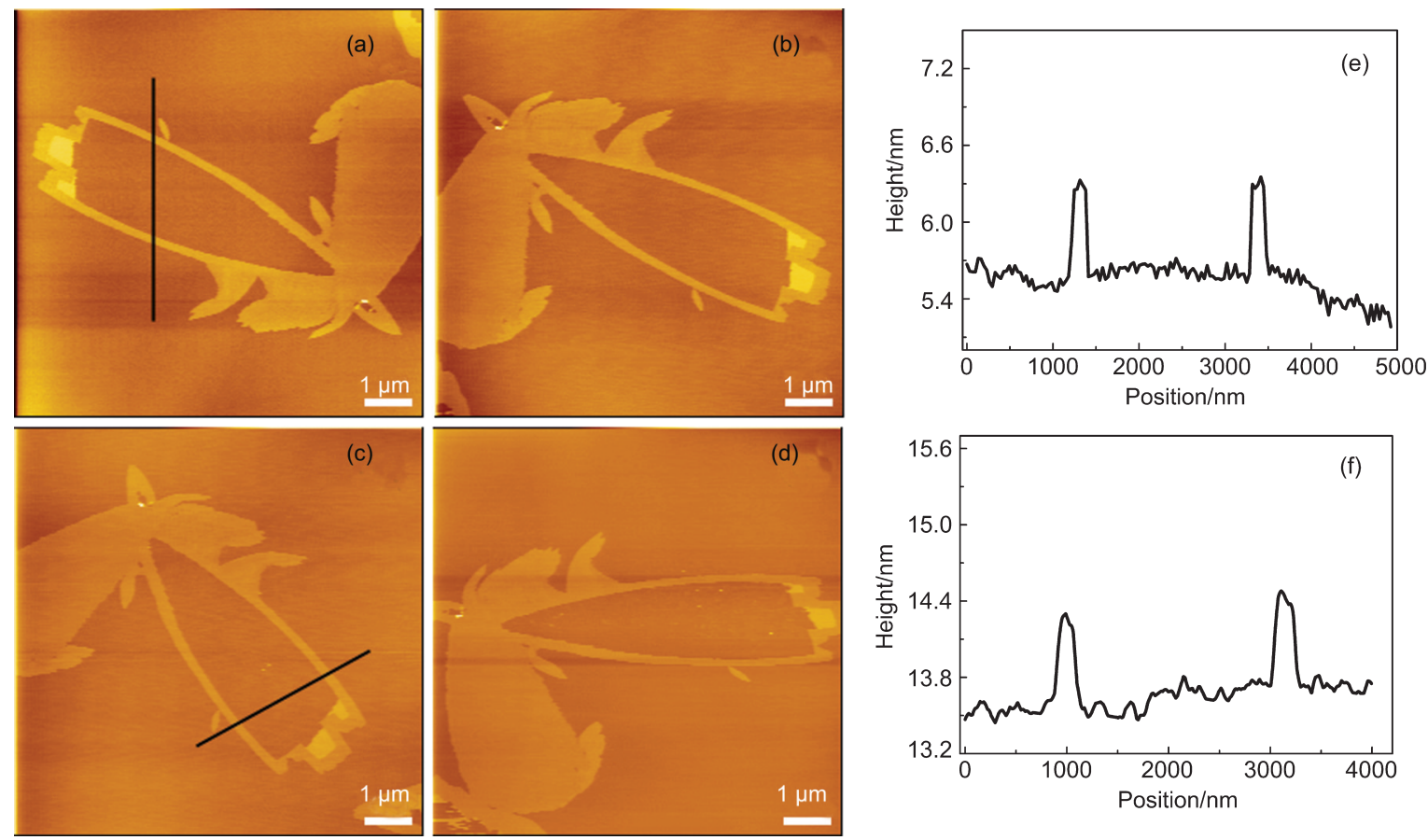

图 4 不同扫描方向石墨烯AFM形貌图

Fig.4 AFM topographic images of graphene at different scanning directions

From (a) to (d), the angles of the AFM topographic images are $90^{\circ},-90^{\circ},-120^{\circ}$, and $-60^{\circ}$, respectively. The corresponding numbers of scans are $22,23,27$, and 33, respectively. (e) and (f) are the line profiles of the marking parts in (a) and (c), respectively.

使在不同的针尖相对运动的方向上, 2 ML 的石墨烯 始终具有相当好的耐磨性.

由于摩擦力与表面的粘附力有着直接的关系, 因此, 我们对不同样品进行了粘附力的研究. 图 5 给 出的是大气环境(图中以 $\mathrm{AE}$ 表示)和真空中(以 $\mathrm{VC}$ 表示)不同厚度石墨烯和云母基底的表面粘附力的 变化情况. 云母基底不同于 $\mathrm{SiO}_{2}$ 基底, 它更容易吸 附水分子及杂质, 但是在真空中这种效应会大大弱 化. ${ }^{25}$ 从图中可见, 大气的云母表面粘附力可达 35

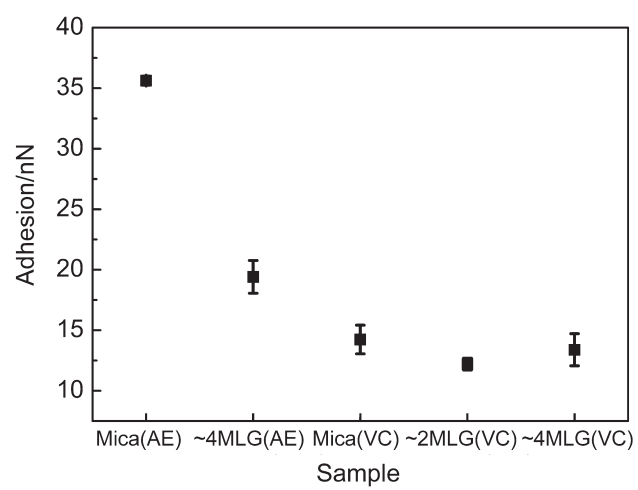

图 5 大气和真空中的石墨烯和云母的粘附力

Fig.5 Adhesion for graphene and mica under the vacuum condition and the ambient environment AE: ambient environment, VC: vacuum condition, 2MLG: 2-ML graphene, 4MLG: 4-ML graphene
$\mathrm{nN}$, 而在真空中可降到 $15 \mathrm{nN}$ 左右. 真空下 2 ML石 墨烯( 2 MLG)与 4 ML 石墨烯( 4 MLG)几乎不可 区分. 由此可见, 粘附力并非是影响不同石墨烯层 数变化导致磨损性质不同的主要原因.

经过采用力曲线的方法多次测量样品表面粘 附力, 发现表面出现了一些亮的点(图 6). 我们推测, 这些高出表面的亮点应该就是粘在针尖表面的石 墨烯碎片, 说明前述磨损实验中被破坏掉结构的石 墨烯碎片吸附在针尖的表面, 但它们与针尖之间的 相互作用并不是特别的强, 在测量力曲线的过程

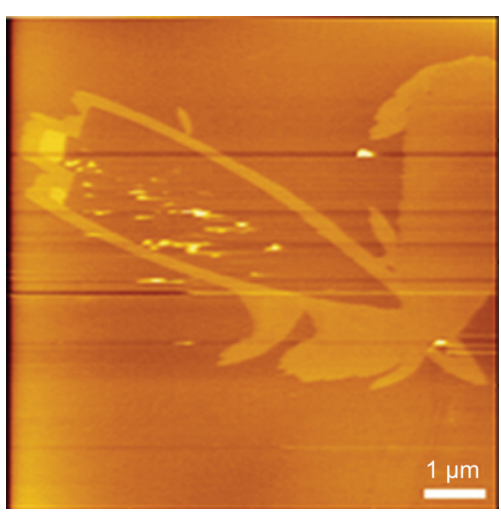

图 6 石墨烯碎片散落在样品表面的 AFM形貌图

Fig.6 AFM topographic image of graphene powder on the surface of the sample 
中, 容易出现脱落的现象, 这也是为什么前面的磨 损图(图 3)上并未在磨损发生点附近观察到碎片石 墨烯的缘故. 有关这方面的研究今后还将进一步深 入.

\section{4 结 论}

(1) HOPG 片层置于无水乙醇中, 控制超声的功 率和溶剂温度, 一定时间后, 离心并取上层清液滴 到干净的云母表面, 可得到不同层数石墨烯, 为进 一步研究石墨烯的磨损性能提供了条件.

(2) 真空条件下, 发现石墨烯薄层的摩擦力和摩 擦系数都随着层数的增加而减小, 到 7 ML之后摩 擦力在一定误差范围内不再变化, 其摩擦系数趋近 于零.

(3) 在磨损实验中数次扫描且改变扫描方向后, 沉积在云母基底上的 2 ML 石墨烯比 4 ML石墨烯 具有更好的耐磨性质. 这可能是因为随着石墨烯的 层数的增加, 其与基底的相互作用力逐步减弱, 也 就更容易被磨掉的原因。

\section{References}

(1) Novoselov, K. S.; Geim, A. K.; Morozov, S. V.; Jiang, D.; Zhang, Y.; Dubonos, S. V.; Grigorieva, I. V.; Firsov, A. A. Science 2004, 306, 666. doi: 10.1126/science. 1102896

(2) Castro Neto, A. H.; Guinea, F.; Peres, N. M. R.; Novoselov, K. S.; Geim, A. K. Rev. Mod. Phys. 2009, 81, 109. doi: 10.1103/ RevModPhys.81.109

(3) Balandin, A. A.; Ghosh, S.; Bao, W. Z.; Calizo, I.; Teweldebrhan, D.; Miao, F.; Lau, C. N. Nano Lett. 2008, 8 (3), 902. doi: $10.1021 / \mathrm{n} 10731872$

(4) Geim, A. K.; Novoselov, K. S. Nat. Mater. 2007, 6, 183. doi: $10.1038 /$ nmat1849

(5) Geim, A. K. Science 2009, 324, 1530. doi: 10.1126/science. 1158877

(6) Novoselov, K. S.; Geim, A. K.; Morozov, S. V.; Jiang, D.; Katsnelson, M. I.; Grigorieva, I. V.; Dubonos, S. V.; Firsov, A. A. Nature 2005, 438, 197. doi: 10.1038/nature04233

(7) Hummers, W. S.; Offeman, R. E. J. Am. Chem. Soc. 1958, 80 (6), 1339. doi: 10.1021/ja01539a017

(8) Hamilton, C. E.; Lomeda, J. R.; Sun, Z.; Tour, J. M.; Barron, A. R. Nano Lett. 2009, 9 (10), 3460. doi: 10.1021/n19016623
(9) Renia, A.; Jia, X. T.; Ho, J.; Nezich, D.; Son, H.; Bulovic, V.; Dresselhaus, M. S.; Kong, J. Nano Lett. 2009, 9 (1), 30. doi: $10.1021 / \mathrm{n} 1801827 \mathrm{v}$

(10) Obraztsov, A. N. Nat. Nanotechnol. 2009, 4, 212. doi: 10.1038/ nnano.2009.67

(11) Berger, C.; Song, Z.; Li, X.; Wu, X.; Brown, N.; Naud, C.; Mayou, D.; Li, T.; Hass, J.; Marchenkov, A. N.; Conrad, E. H.; First, P. N.; de Heer, W. A. Science 2006, 312, 1191. doi: $10.1126 /$ science. 1125925

(12) Donnet, C., Erdemir, A. Surf. Coat. Tech. 2004, 180-181, 76.

(13) Lee, C.; Li, Q.; Kalb, W.; Liu, X. Z.; Berger, H.; Carpick, R. W.; Hone, J. Science 2010, 328, 76. doi: 10.1126/science.1184167

(14) Filleter, T.; McChesney, J. L.; Bostwick, A.; Rotenberg, E.; Emtsev, K. V.; Seyller, T.; Horn, K.; Bennewitz, R. Phys. Rev. Lett. 2009, 102, 086102. doi: 10.1103/PhysRevLett.102.086102

(15) Kim, K. S.; Lee, H. J.; Lee, C.; Lee, S. K.; Jang, H.; Ahn, J. H.; Kim, J. H.; Lee, H. J. ACS Nano 2011, 5, 5107. doi: 10.1021/ nn2011865

(16) Shin, Y. J.; Stromberg, R.; Nay, R.; Huang, H.; Wee, A. T. S.; Yang, H.; Bhatia, C. S. Carbon 2011, 49, 4070. doi: 10.1016/ j.carbon.2011.05.046

(17) Lin, L. Y.; Kim, D. E.; Kim, W. K.; Jun, S. C. Surf. Coat. Tech. 2011, 205, 4864. doi: 10.1016/j.surfcoat.2011.04.092

(18) Sandoz-Rosado, E. J.; Tertuliano, O. A.; Terrell, E. J. Carbon 2012, 50, 4078. doi: 10.1016/j.carbon.2012.04.055

(19) Marchetto, D.; Held, C.; Hausen, F.; Wählisch, F.; Dienwiebel, M.; Bennewitz, R. Tribol. Lett. 2012, 48, 77. doi: 10.1007/ s11249-012-9945-4

(20) Ferrari, A. C.; Meyer, J. C.; Scardaci, V.; Casiraghi, C.; Lazzeri, M.; Mauri, F.; Piscanec, S.; Jiang, D.; Novoselov, K. S.; Roth, S.; Geim, A. K. Phys. Rev. Lett. 2006, 97, 187401. doi: 10.1103/ PhysRevLett.97.187401

(21) Lui, C. H.; Liu, L.; Mak, K. F.; Flynn, G. W.; Heinz, T. F. Nature 2009, 462, 339. doi: 10.1038/nature08569

(22) Li, Q. Y.; Lee, C. G.; Carpick, R. W.; Hone, J. Phys. Status Solidi B 2010, 247, 2909. doi: 10.1002/pssb.v247.11/12

(23) Du, X. Q.; Li, H. Q.; Zhu, Q. R.; Zou, Z. Q.; Liang, Q. Acta Phys. -Chim. Sin. 2011, 27 (10), 2457. [杜晓青, 李慧琴, 朱齐荣, 邹志强, 梁 齐. 物理化学学报, 2011, 27 (10), 2457.] doi: 10.3866/PKU.WHXB20111010

(24) Liang, Q.; Li, H. N.; Xu, Y. B.; Xiao, X. D. J. Phys. Chem. B 2006, 110, 403. doi: 10.1021/jp054939o

(25) Shin, Y. J.; Wang, Y.; Huang, H.; Kalon, G.; Wee, A. T. S.; Shen, Z. X.; Bhatia, C. S.; Yang, H. Langmuir 2010, 26 (6), 3798. doi: $10.1021 / 1 \mathrm{a} 100231 \mathrm{u}$ 